\title{
Adaptabilidade e estabilidade de cultivares registradas de feijão em diferentes épocas de semeadura para a depressão central do Rio Grande do Sul
}

\author{
Adaptability and stability of registred bean cultivars in different growing seasons to central \\ depression region of Rio Grande do Sul
}

Nerinéia Dalfollo Ribeiro ${ }^{1}$ Evandro Jost ${ }^{2}$ Sandro Borba Possebon ${ }^{2}$ Alberto Cargnelutti Filho ${ }^{3}$

RESUMO

O objetivo deste trabalho foi identificar cultivares de feijão com adaptação geral ou específica ao cultivo em diferentes épocas de semeadura e com estabilidade de produção na região da depressão central do Rio Grande do Sul, principalmente, para fins de melhoramento. Os experimentos foram conduzidos no Campus da Universidade Federal de Santa Maria, durante os anos agrícolas 2000/01, 2001/02 e 2002/03, nos cultivos de safra (ambientes favoráveis) e de safrinha (ambientes desfavoráveis). $O$ delineamento experimental utilizado foi o de blocos ao acaso, com três repetições, e avaliaram-se 16 cultivares registradas de feijão. As cultivares Carioca, Iapar 31, Minuano, PR 468 e TPS Bionobre foram identificadas com rendimento de grãos acima da média geral, alta adaptação e estabilidade de produção. A TPS Nobre apresentou adaptabilidade ao cultivo de safra e, para a semeadura no período de safrinha, a Iraí e a Pérola apresentaram alta adaptabilidade.

Palavras-chave: Phaseolus vulgaris L., rendimento de grãos, interação genótipo versus ambiente.

\section{ABSTRACT}

The objective this work was to identify beans cultivars with broad or specific adaptability in different planting times and with yield stability in the central depression region of Rio Grande do Sul to guide breeding programs. The experiments were conducted in the area of the Plant Science Department at the Santa Maria Federal University in three agricultural years in two traditional cropping seasons: rainy (September-October, favorable environment) and dry (January-February, unfavorable environment). A complete randomized experimental blocks design with three replications and 16 registred common bean cultivars were used. Carioca, Iapar 31, Minuano, PR 468 e TPS Bionobre cultivars were identified with mean yield compared to the overall yield, broad adaptability and high stability. The TPS Nobre showed specific adaptability to rainy growing season and Iraí and Pérola showed specific adaptability to dry growing season.

Key words: Phaseolus vulgaris L., grain yield, genotype $x$ environment interaction.

\section{INTRODUÇÃO}

O feijão (Phaseolus vulgaris L.) por ser uma espécie com ciclo anual e desenvolvimento precoce, é mais sensível às variações ambientais (ROSSE \& VENCOVSKY, 2000). Assim, alterações nas condições climáticas podem provocar mudanças acentuadas na produtividade, por isso a identificação de cultivares com adaptação ampla é desejável (RAMALHO et al., 1993).

A diversidade de condições ambientais a que a cultura é submetida contribui para a ocorrência da interação cultivar versus ambiente, ou seja, para a alteração no desempenho relativo das cultivares, em virtude da diferença de ambiente (BORÉM, 1997). O conhecimento dos componentes dessa interação é de grande importância para o melhoramento genético, porém não fornece informações pormenorizadas sobre o comportamento de cada cultivar frente às variações ambientais (CRUZ \& REGAZZI, 1997).

Sendo assim, as análises de adaptabilidade e de estabilidade tornam-se necessárias. Atualmente, há várias metodologias que podem ser utilizadas para a avaliação de um grupo de cultivares numa série de ambientes,

${ }^{1}$ Professor, Doutor, Departamento de Fitotecnia, Centro de Ciências Rurais, Universidade Federal de Santa Maria (UFSM). 97105-900, Santa Maria, RS. E-mail: nerineia@ccr.ufsm.br. Autor para correspondência.

${ }^{2}$ Aluno do Curso de Agronomia, UFSM. Bolsista do Programa Institucional de Bolsas de Iniciação Científica (PIBIC), Conselho Nacional de Desenvolvimento Científico e Tecnológico (CNPq) e Programa Especial de treinamento (PET), Sesu, respectivamente.

${ }^{3}$ Pesquisador em Estatística/Experimentação Agropecuária, Fundação Estadual de Pesquisa Agropecuária (FEPAGRO), Doutor. Rua Gonçalves Dias, 570, Bairro Menino Deus, 90130-060. Porto Alegre, RS. E-mail: alberto-cargnelutti@fepagro.rs.gov.br 
podendo ser citadas as que utilizam regressão linear (FINLAY \& WILKINSON, 1963; EBERHART \& RUSSELL, 1966) ou equação bissegmentada (SILVA \& BARRETO, 1985; PEIXOTO et al., 1985; CRUZ et al., 1989).

Dentre as metodologias que utilizam regressão linear, a utilização do método proposto por EBERHART \& RUSSEL (1966) tem possibilitado a identificação de cultivares de feijão com comportamento previsível em vários Estados do país (SANTOS et al., 1982; DUARTE \& ZIMMERMANN, 1992; MIRANDA et al., 1993; COSTA et al., 1997; COIMBRA et al., 1999a; COIMBRA et al., 1999b; JOBIM et al., 1999a; JOBIM et al., 1999b; JOBIM et al., 2000). Além disso, é o método mais indicado quando o número de ambientes considerados é restrito (VENCOVSKY \& BARRIGA, 1992) e apresenta maior rigor de seleção e de discriminação do tipo de adaptação da cultivar (JOBIM et al., 1999a).

O processo em si está baseado numa análise de regressão linear, no qual o índice ambiental é a variável independente, e a produção média de cada cultivar, em cada ambiente, é a variável dependente (EBERHART \& RUSSEL, 1966). Para esses autores, uma cultivar ideal apresenta produção média alta, coeficiente de regressão igual à unidade, e com desvio de regressão o menor possível $\left(\mathrm{s}^{2} \mathrm{di}=0\right)$, ou seja, possui um comportamento altamente previsível.

Diante desses fatos, torna-se importante o conhecimento do comportamento (adaptabilidade e estabilidade) das cultivares de feijão frente às variações ambientais entre e dentro de anos (safra e safrinha), o que é pouco conhecido nas condições da região da depressão central do Rio Grande do Sul (DC-RS). Essas informações tornam-se indispensáveis, pois é comum a utilização de duas épocas tradicionais de semeadura de feijão, a safra (setembro a outubro) e a safrinha (janeiro a fevereiro). Por sua vez, para o registro das novas cultivares de feijão, junto ao Serviço Nacional de Proteção de Cultivares, no Ministério da Agricultura (SNPCMA) se considera, normalmente, apenas os dados obtidos do cultivo de safra.

O objetivo deste trabalho foi identificar, entre as cultivares registradas de feijão para cultivo no Rio Grande do Sul, as cultivares com adaptação geral ou específica a ambientes favoráveis ou desfavoráveis e com estabilidade de produção para a região da DC-RS, principalmente, para a orientação de programas de melhoramento.

\section{MATERIAL E MÉTODOS}

Os experimentos foram conduzidos nos anos agrícolas 2000/01, 2001/02 e 2002/03, nos cultivos de safra (semeadura em setembro-outubro) e de safrinha (semeadura em janeiro-fevereiro), em solo Alissolo Hipocrômico argilúvico típico, pertencente à unidade de mapeamento Santa Maria. O local de condução foi o Campus da Universidade Federal de Santa Maria, região fisiográfica da depressão central do Rio Grande do Sul, entre as coordenadas geográficas de $29^{\circ} 45^{\prime}$ de latitude sul, $53^{\circ} 42^{\prime}$ de longitude a oeste de Greenwich, e a uma altitude de $95 \mathrm{~m}$.

Os ensaios foram delineados em blocos casualizados, com três repetições, sendo a unidade experimental constituída por quatro fileiras de $4 \mathrm{~m}$ de comprimento, espaçadas em $0,5 \mathrm{~m}$, e com área útil de $3 \mathrm{~m}^{2}$ (foram consideradas apenas as duas fileiras centrais, desprezando-se $0,5 \mathrm{~m}$ nas extremidades). A densidade de semeadura foi ajustada de acordo com o hábito de crescimento de cada cultivar (CEPEF, 2001). Os tratamentos avaliados constituiram de 16 cultivares de feijão registradas para o cultivo no Estado do Rio Grande do Sul (Tabela 1).

O preparo do solo foi realizado de forma convencional e as adubações foram baseadas na análise química do solo. Os tratos culturais - controle de insetos e de plantas invasoras - foram realizados sempre que necessário, a fim de não comprometer o desenvolvimento normal da cultura. Os efeitos de genótipos e de ambientes foram considerados fixos e a análise de adaptabilidade e de estabilidade foi realizada segundo o modelo de EBERHART \& RUSSELL (1966).

\section{RESULTADOS E DISCUSSÃO}

As análises de adaptabilidade e de estabilidade foram realizadas com os dados obtidos para rendimento de grãos na safra $(2000 / 01,2001 / 02$ e 2002/03) e na safrinha (2000/01 e 2001/02) (Tabela 1).

A média obtida para o rendimento de grãos nos ensaios conduzidos na época da safra foi superior à média geral dos ensaios $\left(1488 \mathrm{~kg} \mathrm{ha}^{-1}\right), \mathrm{o}$ que caracteriza ambientes favoráveis (índices ambientais positivos). Por sua vez, os ensaios de safrinha apresentaram média inferior à $1488 \mathrm{~kg} \mathrm{ha}^{-1}$, representando ambientes desfavoráveis (índices ambientais negativos). Sendo assim, é possível investigar a ocorrência de cultivares com adaptação ampla (safra e safrinha) e específica (safra ou safrinha), bem como verificar sua previsibilidade de comportamento. 
Tabela 1 - Rendimento de grãos de cultivares de feijão $\left(\mathrm{kg} \mathrm{ha}^{-1}\right)$ em cinco ambientes, média geral $\left(\mathrm{kg}^{-1}\right)$, coeficiente de variação $(\mathrm{CV} \%)$, quadrado médio do erro (QMe) e índice ambiental (IA). Santa Maria - RS, UFSM, 2003.

\begin{tabular}{|c|c|c|c|c|c|c|c|c|c|c|c|}
\hline \multirow[t]{2}{*}{ Cultivares* } & \multicolumn{10}{|c|}{ Ambientes } & \multirow[t]{2}{*}{ Média } \\
\hline & \multicolumn{2}{|c|}{ Safra 2000/01 } & \multicolumn{2}{|c|}{ Safra 2001/02 } & \multicolumn{2}{|c|}{ Safra 2002/03 } & \multicolumn{2}{|c|}{ Safrinha 2000/01 } & \multicolumn{2}{|c|}{ Safrinha $2001 / 02$} & \\
\hline Carioca & 2063 & $\mathrm{a}$ & 2252 & $\mathrm{a}$ & 2047 & $\mathrm{~b}$ & 841 & $\mathrm{~b}$ & 819 & $\mathrm{a}$ & 1604 \\
\hline Diamante Negro & 1331 & $\mathrm{~b}$ & 2195 & $\mathrm{a}$ & 1173 & $\mathrm{c}$ & 672 & $\mathrm{~b}$ & 656 & $\mathrm{a}$ & 1205 \\
\hline TPS Nobre & 1976 & $\mathrm{a}$ & 2325 & a & 2592 & $\mathrm{a}$ & 757 & $\mathrm{~b}$ & 844 & $\mathrm{a}$ & 1699 \\
\hline Guapo Brilhante & 2471 & $\mathrm{a}$ & 2330 & $\mathrm{a}$ & 3033 & a & 1321 & a & 1027 & $\mathrm{a}$ & 2036 \\
\hline Guateian 6662 & 1480 & $\mathrm{~b}$ & 1988 & a & 1839 & $\mathrm{~b}$ & 781 & $\mathrm{~b}$ & 449 & b & 1307 \\
\hline Iapar 31 & 2094 & $\mathrm{a}$ & 2168 & $\mathrm{a}$ & 1847 & $\mathrm{~b}$ & 991 & $\mathrm{a}$ & 772 & $\mathrm{a}$ & 1575 \\
\hline Iapar 44 & 1353 & $\mathrm{~b}$ & 1861 & $\mathrm{a}$ & 1026 & $\mathrm{c}$ & 514 & $\mathrm{~b}$ & 280 & b & 1007 \\
\hline Iraí & 2104 & $\mathrm{a}$ & 1383 & a & 2148 & $\mathrm{~b}$ & 1423 & $\mathrm{a}$ & 1149 & $\mathrm{a}$ & 1642 \\
\hline Macanudo & 1719 & $\mathrm{~b}$ & 2009 & $\mathrm{a}$ & 2013 & $\mathrm{~b}$ & 988 & $\mathrm{a}$ & 620 & $\mathrm{a}$ & 1470 \\
\hline Macotaço & 2090 & $\mathrm{a}$ & 2081 & $\mathrm{a}$ & 1122 & $\mathrm{c}$ & 1239 & $\mathrm{a}$ & 744 & $\mathrm{a}$ & 1455 \\
\hline Minuano & 2502 & $\mathrm{a}$ & 2494 & $\mathrm{a}$ & 2395 & $\mathrm{a}$ & 1191 & $\mathrm{a}$ & 984 & $\mathrm{a}$ & 1913 \\
\hline Pérola & 1438 & $\mathrm{~b}$ & 1822 & $\mathrm{a}$ & 1034 & $\mathrm{c}$ & 760 & $\mathrm{~b}$ & 718 & $\mathrm{a}$ & 1154 \\
\hline Rio Tibagi & 1619 & $\mathrm{~b}$ & 1668 & $\mathrm{a}$ & 1351 & $\mathrm{c}$ & 672 & $\mathrm{~b}$ & 348 & $\mathrm{~b}$ & 1132 \\
\hline PR 468 & 2377 & $\mathrm{a}$ & 2484 & $\mathrm{a}$ & 1810 & $\mathrm{~b}$ & 1241 & $\mathrm{a}$ & 786 & $\mathrm{a}$ & 1740 \\
\hline TPS Bionobre & 1754 & $\mathrm{~b}$ & 2197 & $\mathrm{a}$ & 2015 & $\mathrm{~b}$ & 925 & $\mathrm{~b}$ & 860 & $\mathrm{a}$ & 1550 \\
\hline TPS Bonito & 1221 & $\mathrm{~b}$ & 1765 & $\mathrm{a}$ & 2421 & $\mathrm{a}$ & 342 & $\mathrm{~b}$ & 875 & $\mathrm{a}$ & 1325 \\
\hline Média & 1850 & & 2064 & & 1867 & & 916 & & 746 & & 1488 \\
\hline CV $(\%)$ & 17,40 & & 19,66 & & 18,27 & & 22,56 & & 24,64 & & \\
\hline QMe & 103544 & & 164637 & & 116255 & & 42734 & & 33760 & & \\
\hline IA & 362 & & 576 & & 379 & & -572 & & -742 & & \\
\hline
\end{tabular}

* Grupos de médias seguidas pela mesma letra não diferem pelo critério de Scott-Knott em nível de 5\% de probabilidade.

A relação entre o maior e o menor quadrado médio do erro dos ambientes $(4,9)$ foi inferior ao valor (7) sugerido por PIMENTEL GOMES (1990), indicando homogeneidade das variâncias residuais entre os ambientes, o que possibilita a realização da análise conjunta. Houve interação cultivar versus ambiente, indicando comportamento diferenciado das cultivares nos diferentes ambientes (Tabela 2). Presença de interações significativas entre cultivares versus anos e cultivares versus épocas de semeadura são relatadas em experimentos com feijão (RAMALHO et al., 1998; COIMBRA et al., 1999b; ELIAS et al., 1999), o que dificulta a seleção de cultivares de feijão capazes de expressar alto potencial de rendimento em condições ambientais diversas (MEKBIB, 2002).

As cultivares de feijão avaliadas que apresentaram coeficiente de regressão igual à unidade têm ampla adaptabilidade a região da Depressão Central do Rio Grande do Sul. Assim, destacaram-se: Carioca, Diamante Negro, Guapo Brilhante, Guateian 6662, Iapar 31, Iapar 44, Macanudo, Macotaço, Minuano, Rio Tibagi, PR 468, TPS Bionobre e TPS Bonito (Tabela 3). Resultado semelhante foi obtido para as cultivares
- Macanudo, Minuano e Macotaço - em um trabalho conduzido durante o período de 1988/89 a 1993/94, em 23 ambientes no Estado do Rio Grande do Sul (PIANA et al., 1999). Em Santa Catarina, 'Macanudo' também apresentou destacada adaptabilidade em cinco anos de avaliação (ELIAS et al., 1999).

Dez cultivares apresentaram desvio de regressão próximo a zero, caracterizando alta estabilidade (Carioca, Guateian 6662, Iapar 31, Iapar 44, Macanudo, Minuano, Pérola, Rio Tibagi, PR 468 e TPS Bionobre). A cultivar Carioca também apresentou alta estabilidade de produção em Minas Gerais (COSTA et al., 1997). No entanto, em trabalho conduzido por JOBIN et al. (1999a), no estado do Rio Grande do Sul, essa cultivar apresentou adaptação ampla e médio rendimento de grãos, quando comparada às demais cultivares de feijão avaliadas em cinco locais, nos anos agrícolas de 1987 e de 1988, pelo modelo de EBERHART \& RUSSELL (1966). Por sua vez, PIANA et al. (1999) não identificaram nenhuma cultivar com estabilidade de produção para o estado do Rio Grande do Sul, pois todas se apresentaram instáveis. Esses resultados 
Tabela 2 - Resumo da análise de variância conjunta do rendimento de grãos $\left(\mathrm{kg} \mathrm{ha}^{-1}\right)$ de 16 cultivares de feijão com a decomposição da soma de quadrados de ambientes/cultivares, segundo Eberhart \& Russell (1966). Santa Maria - RS, UFSM, 2003.

\begin{tabular}{|c|c|c|}
\hline Causas de variação & $\begin{array}{l}\text { Graus de } \\
\text { liberdade }\end{array}$ & Quadrado médio \\
\hline Blocos/Ambientes & 10 & 2478175 \\
\hline Cultivares (C) & 15 & $1248407 *$ \\
\hline Ambientes (A) & 4 & $17805770 *$ \\
\hline Interação CxA & 60 & $267951 *$ \\
\hline Ambientes/Cultivares & 64 & 1364065 \\
\hline Ambiente linear & 1 & $71223078 *$ \\
\hline Cultivar x Ambiente linear & 15 & $222145^{*}$ \\
\hline Dev. combinação & 48 & $265519 *$ \\
\hline \multicolumn{3}{|l|}{ Desvios de regressão } \\
\hline Carioca & 3 & $17622^{\mathrm{ns}}$ \\
\hline Diamante negro & 3 & $415267 *$ \\
\hline TPS nobre & 3 & $263484 *$ \\
\hline Guapo brilhante & 3 & $462282 *$ \\
\hline Guateian 6662 & 3 & $77347^{\mathrm{ns}}$ \\
\hline Iapar 31 & 3 & $36148^{\mathrm{ns}}$ \\
\hline Iapar 44 & 3 & $212359^{\mathrm{ns}}$ \\
\hline Iraí & 3 & $497382 *$ \\
\hline Macanudo & 3 & $59956^{\mathrm{ns}}$ \\
\hline Macotaço & 3 & $620115^{*}$ \\
\hline Minuano & 3 & $37773^{\mathrm{ns}}$ \\
\hline Pérola & 3 & $224489^{\mathrm{ns}}$ \\
\hline Rio Tibagi & 3 & $53782^{\mathrm{ns}}$ \\
\hline PR 468 & 3 & $223875^{\mathrm{ns}}$ \\
\hline TPS Bionobre & 3 & $42851^{\mathrm{ns}}$ \\
\hline TPS Bonito & 3 & $1003566 *$ \\
\hline Resíduo & 150 & 92186 \\
\hline
\end{tabular}

* Significativo a $5 \%$ de probabilidade pelo teste $\mathrm{F}$; ${ }^{\text {ns }}$ não significativo.

destacam o efeito da interação cultivares versus ambientes e a importância da realização dos ensaios em maior número de anos e de épocas de semeadura, com já sugerido por RAMALHO et al. (1998), a fim de que seja possível a identificação de cultivares de feijão com adaptabilidade e/ou estabilidade de produção para uma determinada região de cultivo.

As cultivares - Diamante Negro, TPS Nobre, Guapo Brilhante, Iraí, Macotaço e TPS Bonito - apresentaram significância dos desvios de regressão $\left(\sigma_{\mathrm{di}}^{2} \neq 0\right)$, ou seja, baixa previsibilidade de comportamento quanto a suas respostas às variações ambientais. No entanto, o grau de imprevisibilidade não deve comprometer a indicação daquelas cultivares com $\mathrm{R}^{2}$ elevado.

A 'Guapo Brilhante' pode ser considerada promissora, pois além de apresentar a maior média de rendimento de grãos, tem ampla adaptabilidade. Porém sua desvantagem é de apresentar baixa previsibilidade de comportamento. Entretanto esta cultivar não deve ser julgada totalmente indesejável, uma vez que seu $\mathrm{R}^{2}$ atingiu nível de $83,49 \%$.

A 'TPS Nobre' apresentou média de rendimento de grãos acima da média geral e adaptabilidade específica aos ambientes favoráveis (safra), porém baixa previsibilidade de comportamento, mas com $\mathrm{R}^{2}$ de $90,87 \%$. Por outro lado, 'Iraí' e 'Pérola' apresentaram adaptabilidade específica aos ambientes desfavoráveis (safrinha), sendo que a 'Iraî', apesar de apresentar média de rendimento de grãos superior à média geral, é imprevisível e tem $\mathrm{R}^{2}$ baixo; enquanto que a 'Pérola' obteve rendimento de grãos inferior à média geral, mas possui alta previsibilidade de comportamento. Entretanto, era de se esperar que a Iraí apresentasse comportamento mais instável do que as demais cultivares avaliadas, haja vista que seu hábito de crescimento é determinado (planta tipo I) e ciclo biológico curto, o que contribui para uma maior suscetibilidade ao estresse ambiental (PIANA et al., 1999).

As cultivares - Carioca, Iapar 31, Minuano, PR 468 e TPS Bionobre - podem ser consideradas como desejáveis, segundo os critérios de EBERHART \& RUSSELL (1966), ou seja, apresentam rendimento de grãos acima da média geral $\left(1488 \mathrm{~kg} \mathrm{ha}^{-1}\right)$, coeficiente de regressão igual a 1 (ampla adaptabilidade) e desvios da regressão igual a zero (estabilidade alta).

A seleção para produção de grãos é mais fácil do que para a estabilidade de produção (SANTOS et al., 1982). O melhoramento para estabilidade de produção é complexo, pois envolve mecanismos genéticos, fisiológicos e morfológicos (MEKBIB, 2002). Por isso, espera-se dificuldades na seleção de cultivares de feijão com alto rendimento de grãos, estabilidade de produção e tipo de grão adequado às exigências de mercado. No presente trabalho, considerando-se os diferentes grupos comerciais, apenas TPS Bionobre (preto), Carioca (carioca) e PR 468 (manteigão) apresentam características de grão adequadas à preferência dos consumidores. A Iapar 31 e a Minuano, apesar de apresentarem comportamento desejável, segundo os critérios de EBERHART \& RUSSEL (1966), apresentam restrições para a comercialização por apresentarem halo amarelo e grãos arroxeados, respectivamente.

Sendo assim, apesar de não haver a necessidade de informar a adaptabilidade e a 
Tabela 3 - Estimativas dos parâmetros de adaptabilidade e de estabilidade fenotípica do rendimento de grãos, de 16 cultivares de feijão, avaliadas em cinco ambientes, segundo a metodologia de Eberhart \& Russell (1966). Santa Maria - RS, UFSM, 2003.

\begin{tabular}{|c|c|c|c|c|}
\hline Cultivar & Média de rendimento $\left(\mathrm{kg} \mathrm{ha}^{-1}\right)$ & Coeficiente de regressão $\left(\hat{\beta}_{1 \mathrm{i}}\right)$ & Desvio de regressão ( $\hat{\sigma}_{\mathrm{di}}^{2}$ ) & $\mathrm{R}_{\mathrm{i}}^{2}(\%)$ \\
\hline Carioca & 1604 & $1,163^{\mathrm{ns}}$ & $-24855^{\mathrm{ns}}$ & 99,13 \\
\hline Diamante Negro & 1205 & $0,887^{\mathrm{ns}}$ & $107694 *$ & 73,76 \\
\hline TPS Nobre & 1699 & $1,329 *$ & $57100 *$ & 90,87 \\
\hline Guapo Brilhante & 2036 & $1,255^{\mathrm{ns}}$ & $123366^{*}$ & 83,49 \\
\hline Guateian 6662 & 1307 & $1,074^{\mathrm{ns}}$ & $-4946^{\mathrm{ns}}$ & 95,68 \\
\hline Iapar 31 & 1575 & $1,053^{\mathrm{ns}}$ & $-18679^{\mathrm{ns}}$ & 97,85 \\
\hline Iapar 44 & 1007 & $0,974^{\mathrm{ns}}$ & $40058^{\mathrm{ns}}$ & 86,90 \\
\hline Iraí & 1642 & $0,472 *$ & $135065^{*}$ & 39,93 \\
\hline Macanudo & 1470 & $1,019^{\mathrm{ns}}$ & $-10743^{\mathrm{ns}}$ & 96,26 \\
\hline Macotaço & 1455 & $0,752^{\mathrm{ns}}$ & $175976^{*}$ & 57,50 \\
\hline Minuano & 1913 & $1,235^{\mathrm{ns}}$ & $-18138^{\mathrm{ns}}$ & 98,36 \\
\hline Pérola & 1154 & $0,668 *$ & $44101^{\mathrm{ns}}$ & 74,67 \\
\hline Rio Tibagi & 1132 & $0,952^{\mathrm{ns}}$ & $-12801^{\mathrm{ns}}$ & 96,15 \\
\hline PR 468 & 1740 & $1,132^{\mathrm{ns}}$ & $43896^{\mathrm{ns}}$ & 89,46 \\
\hline TPS Bionobre & 1550 & $1,005^{\mathrm{ns}}$ & $-16445^{\mathrm{ns}}$ & 97,22 \\
\hline TPS Bonito & 1325 & $1,029^{\mathrm{ns}}$ & $303793 *$ & 61,02 \\
\hline
\end{tabular}

$\mathrm{H}_{0}=\hat{\beta}_{1 \mathrm{i}}=1 *$ significativo a $5 \%$ de probabilidade pelo teste $\mathrm{t}$; ${ }^{\text {ns }}$ não significativo.

$\mathrm{H}_{0}=\hat{\sigma}_{\mathrm{di}}^{2}=0 *$ significativo a $5 \%$ de probabilidade pelo teste $\mathrm{F} ;{ }^{\mathrm{ns}}$ não significativo.

estabilidade de produção para o registro das novas cultivares de feijão, junto ao Serviço Nacional de Proteção de Cultivares (SNPC- Brasília), essas informações continuam a ser necessárias para o conhecimento da base genética do germoplasma disponível e maior discernimento entre as cultivares. Talvez, a simplificação da norma para a inscrição das novas cultivares, tenha trazido, como conseqüência, o menor conhecimento do germoplasma.

O grande desafio dos programas de melhoramento da atualidade é o desenvolvimento de cultivares de feijão com elevado potencial de rendimento de grãos, estabilidade de produção e com grãos adequados à preferência dos consumidores.

\section{CONCLUSÕES}

Com base nos resultados apresentados, e para os ambientes considerados, pode-se concluir que, para a região da depressão central do Rio Grande do Sul, as condições ambientais influenciam na capacidade de adaptação e de estabilidade de produção das cultivares de feijão. As cultivares - Carioca, Iapar 31, Minuano, PR 468 e TPS Bionobre - apresentam alta adaptação e estabilidade de produção. A TPS Nobre apresenta adaptação específica para o cultivo de safra e a Iraí e a Pérola são adaptadas para a semeadura de safrinha.

\section{REFERÊNCIAS BIBLIOGRÁFICAS}

BORÉM, A. Melhoramento de plantas. Viçosa : UFV, 1997. 547p.

CEPEF. Feijão: recomendações técnicas para o cultivo de feijão no Rio Grande do Sul. Erechim : São Cristovão, 2001. 112p.

COIMBRA, J.L.M. et al. Adaptabilidade e estabilidade de fenótipos em genótipos de feijão de cor (Phaseolus vulgaris.L.) em três ambientes distintos. Ciência Rural, Santa Maria, v.29, n.3, p.441-448, 1999a.

COIMBRA, J.L.M. et al. Reflexos da interação genótipo x ambiente e suas implicações nos ganhos de seleção em genótipos de feijão (Phaseolus vulgaris.L.). Ciência Rural, Santa Maria, v.29, n.3, p.433-439, 1999b.

COSTA, A.S.V. et al. Comportamento de cultivares de feijão (Phaseolus vulgaris L.) em dez ambientes compreendendo cinco sistemas de produção. Revista Ceres, Viçosa, v.44, n.256, p.676-700, 1997.

CRUZ, C.D.; REGAZZI, A.J. Modelos biométricos aplicados ao melhoramento genético. 2.ed. Viçosa: UFV, 1997. 390p.

CRUZ, C.D.; TORRES, R.A.A.; VECOVSKY, R. An alternative approach to the stability analysis proposed by Silva and

Ciência Rural, v.34, n.5, set-out, 2004. 
Barreto. Revista Brasileira de Genética, Ribeirão Preto, v.12, n.2, p.567-580, 1989.

DUARTE, J.B.; ZIMMERMANN, M.J.O. Comparison of three methods used for the study of adaptation and phenotypic stability $\mathrm{n}$ the common bean (Phaseolus vulgaris L.). Brazilian Journal of Genetics, Ribeirão Preto, v.15, n.1, p.125-136, 1992.

EBERHART, S.A.; RUSSELL, W.A. Stability parameters for comparing varieties. Crop Science, Madison, v.6, n.1, p.3640, 1966.

ELIAS, H. T.; HEMP, S.; CANTON, T. Análise da interação genótipo $\mathrm{x}$ ambiente na avaliação de cultivares de feijão em Santa Catarina. Pesquisa Agropecuária Gaúcha, Porto Alegre, v.5, n.2, p.271-275, 1999.

FINLAY, K.W.; WILKINSON, G.N. The analysis of adaptation in a plant breeding programme. Australian Journal of Agricultural Research, Collingwood, v. 14, p.742-754, 1963.

JOBIM, C.I.P.; WESTPHALEN, S.L.; FEDERIZZI, L.C. Análise da interação genótipo x ambiente para o rendimento de grãos em feijão. Pesquisa Agropecuária Gaúcha, Porto Alegre, v.5, n.2, p. 161-171, 1999a.

JOBIM, C.I.P.; WESTPHALEN, S.L.; FEDERIZZI, L.C. Utilização de variáveis ambientais e índice de doença na análise da interação genótipo $\mathrm{x}$ ambiente em feijão. Pesquisa Agropecuária Gaúcha, Porto Alegre, v.5, n.2, p. 173-182, 1999b.

JOBIM, C.I.P. et al. Contribuição de variáveis ambientais à interação genótipo $x$ ambiente em feijão. Pesquisa Agropecuária Gaúcha, Porto Alegre, v.6, n.1, p. 27-38, 2000 .

MEKBIB, F. Simultaneous selection for high yield and stability in common bean (Phaseolus vulgaris L.) gotypes. Journal of Agricultural Science, Cambridge, v.138, n3, p.249-253, 2002 .

MIRANDA, V.M. et al. Adaptabilidade e estabilidade de comportamento de cultivares de feijão em quatro municípios da Zona da Mata de Minas Gerais. Revista Ceres, Viçosa, v.41, n.232, p.591-609, 1993.
PEIXOTO, T.C.; SILVA, J.G.C; BARRETO, J.N. Técnicas de análise de interação genótipo por ambiente e estabilidade de clones de cana-de-açúcar. In: SIMPÓSIO DE ESTATÍSTICA APLICADA À EXPERIMENTAÇÃO AGRONÔMICA, 1., 1985, Campinas, SP. Proceedings... Campinas : Cargill, 1985. p.243-258.

PIANA, C.F.B. et al. Adaptabilidade e estabilidade do rendimento de grãos de genótipos de feijão. Pesquisa Agropecuária Brasileira, Brasília, v.34, n.4, p.553-564, 1999.

PIMENTEL GOMES, F. Curso de estatística experimental. 13.ed. Piracicaba : Livraria Nobel, 1990. $467 \mathrm{p}$.

RAMALHO, M.A.; SANTOS, J.B.; ZIMMERMANN, M.J.O. Genética quantitativa em plantas autógamas: aplicações ao melhoramento do feijoeiro. Goiânia : UFG, 1993. 271p.

RAMALHO, M.A.P.; ABREU, A.F.B.; SANTOS, P.S.J. Interações genótipos $\mathrm{x}$ épocas de semeadura, anos e locais na avaliação de cultivares de feijão nas regiões sul e Alto Paranaíba em Minas Gerais. Ciência e Agrotecnica, Lavras, v.22, n.2, p.176-181, 1998.

ROSSE, L.N.; VENCOVSKY, R. Modelo de regressão nãolinear aplicado ao estudo da estabilidade fenotípica de genótipos de feijão no estado do Paraná. Bragantia, Campinas, v.59, n.1, p.99-107, 2000.

SANTOS, J.B.; VELO, N.A.; RAMALHO, A.P. Stability of grain yield and of its basic components in beans (Phaseolus vulgaris L.). Revista Brasileira de Genética, Ribeirão Preto, v,4, p.761-772, 1982.

SILVA, J.G.C.; BARRETO, J.N. Aplicação de regressão linear segmentada em estudos da interação genótipo $\mathrm{x}$ ambiente. In: SIMPÓSIO DE ESTATÍSTICA APLICADA À EXPERIMENTAÇÃO AGRONÔMICA, 1., 1985, Campinas, SP. Proceedings... Campinas : Cargill, 1985. p.49-50.

VENCOVSKY, R.; BARRIGA, P. Genética biométrica no fitomelhoramento. Ribeirão Preto : Sociedade Brasileira de Genética, 1992. 486 p. 